\title{
Mental rotation of faces
}

\author{
TIM VALENTINE \\ MRC Applied Psychology Unit, Cambridge, England \\ and \\ VICKI BRUCE \\ University of Nottingham, Nottingham, England
}

\begin{abstract}
The effect of orientation upon face recognition was explored in two experiments, which used a procedure adapted from the mental rotation literature. In the first experiment, a linear increase in the RT of same-different judgments was found as the second of a pair of sequentially presented faces was rotated away from the vertical. Also, it was found that the effect of changing facial expression did not interact with orientation. In the second experiment, a linear relationship between RT and orientation was found in a task involving the recognition of famous faces. This recognition task was found to be more affected by inversion than was an expression classification task. These results are interpreted as evidence against the view that inverted faces are processed in a qualitatively different manner from upright faces, and are also inconsistent with the hypothesis that inversion makes faces difficult to recognize because facial expression cannot be extracted from an inverted face.
\end{abstract}

Upside-down faces are known to be difficult to recognize. The recognition of faces is more disrupted by stimulus inversion than is the recognition of other classes of visual objects, for example, houses, dogs' faces, and landscapes (Diamond \& Carey, 1986; Scapinello \& Yarmey, 1970; Valentine \& Bruce, 1986; Yarmey, 1971; Yin, 1969). The effect of inversion upon face recognition is of some theoretical significance because it has been cited as one of several lines of evidence that suggest that face recognition may be in some way special, possibly involving a process that is not involved in the recognition of other visual objects (e.g., Yin, 1969, 1970). The disproportionate effect of inversion upon face recognition may be due to the disruption of some process that has developed for the highly overlearned skill of recognizing upright faces.

Several different but related hypotheses have been proposed regarding the specific nature of such a process. The first hypothesis is based on a distinction between two types of information available for the recognition of upright faces, information about the individual features (component or piecemeal information) and information of the spatial relationships between individual features (configural information). Carey and Diamond (1977) proposed that the disproportionate effect of inversion upon face recognition arises because inversion is particularly disruptive to the encoding of configural information. Thus, when required to recognize an upright face, subjects can rely

\footnotetext{
We thank Sam Grainger for the photographic work involved in producing the stimuli and Ian Nimmo-Smith for statistical advice. The experiments described here were conducted as part of the first author's research for his doctorate. We also thank the Economic and Social Research Council for grants, which helped support this work. Tim Valentine's mailing address is MRC Applied Psychology Unit, 15 Chaucer Road, Cambridge $\mathrm{CB} 22 \mathrm{EF}$, England.
}

on both component and configural information, but when required to recognize an inverted face, they are forced to rely on component information alone.

Rock (1973) proposed a similar explanation of the difficulty in recognizing inverted faces. He suggested that faces overtax a system for rotating disoriented stimuli. Rock argues that the inability to correct the orientation of all the features simultaneously makes inverted faces difficult to recognize. Because the features need to be rotated one at a time, information about the spatial relationships between the features (configural information) cannot be recovered.

Diamond and Carey (1986) presented further evidence related to the hypothesis that configural information cannot be extracted from an inverted face. They examined the role of expertise in the effect of inversion and found that dog experts showed an effect of inversion on their recognition of individual dogs, which was comparable to the observed effect of inversion on their recognition of human faces. Novices, however, showed the usual materials $x$ orientation interaction. Their recognition of faces was more impaired by inversion than was their recognition of dogs. Diamond and Carey suggest that a large effect of inversion will be observed when three conditions are met: (1) that the recognition task involves the discrimination of a class of stimuli that share a common configuration; (2) that the members of the class can be differentiated on the basis of distinctive relations among the elements that define the common configuration (termed second-order relational properties); and (3) that the subjects have the expertise to make use of these distinctive relations. Diamond and Carey argue that faces form a highly homogeneous class of stimuli of which many exemplars are experienced and, therefore, are represented in terms of second-order relational information. Follow- 
ing Carey and Diamond's (1977) hypothesis, they argue that the ability to encode second-order relational information is particularly sensitive to inversion. Therefore, the recognition of inverted faces must be achieved on the basis of encoding isolated features. Diamond and Carey's study is important because it demonstrates that a large effect of inversion is not specific to face recognition. However, it raises questions about the nature and role of second-order relational information in recognizing faces.

Carey and Diamond's (1977) hypothesis that inversion disrupts the encoding of configural information, is based on the assumption that there is a qualitative difference in the processing of upright and inverted faces. Qualified support for this assumption has come from studies that have shown a reduced influence of configural information in inverted faces (e.g., Maruyama \& Endo, 1983, 1984); only one study (Sergent, 1984) has shown a qualitative difference in the processing of upright and inverted faces. Using a multidimensional scaling technique, Sergent found evidence that both component and configural information were used in a simultaneous matching task in which upright Photo-Fit faces served as stimuli. However, when the stimuli were inverted, there was no evidence that subjects made use of configural information. Sergent's study provides clear evidence of a qualitative difference in the processing of upright and inverted face stimuli. However, the highly artificial stimuli used and the manipulation of the differences between them make it difficult to generalize from this result to the recognition of real faces.

Although Carey and Diamond (1977) postulate a qualitative difference in the processing of upright and inverted faces, it is possible to argue that inversion causes a quantitative but not a qualitative difference in face processing. Inversion undoubtably makes the encoding of configural information slower and less accurate but might not make it impossible. The essential difference between this variation of Carey and Diamond's hypothesis and their view is the question of whether recognition of inverted faces depends upon different features from the recognition of upright faces.

A second hypothesis, proposed by Goldstein and Chance (1980), posits that the disproportionate effect of inversion upon face recognition arises from the development of a face schema. Because the recognition of upright faces is a highly practiced skill, the face schema has become highly developed. Goldstein and Chance argue that although this schema increases the efficiency with which upright faces can be processed, it becomes increasingly rigid with development and therefore becomes relatively poor at processing unusual stimuli, such as inverted faces. Although Goldstein and Chance do not elaborate upon the actual process by which a schema is used, unlike Carey and Diamond's (1977) hypothesis, schema theory does not necessarily postulate a switch in strategy for processing inverted stimuli.

A third hypothesis for the disproportionate effect of inversion upon face recognition is that upside-down faces may be difficult to recognize because inversion may dis- rupt the interpretation of facial expression (Yin, 1970). It is possible, of course, that analysis of facial expression is an example of a task that relies heavily upon the processing of second-order relational information. In this respect, Yin's hypothesis may also be regarded as having much in common with Carey and Diamond's piecemeal-configural distinction. However, Yin's hypothesis differs in one important respect: It implies that the recognition of facial identity is dependent upon the processing of facial expression.

The "Margaret Thatcher illusion" provides a powerful demonstration of the effect of inversion upon the recognition of expression (Parks, Coss, \& Coss, 1985; Thompson, 1980; Valentine \& Bruce, 1985). In this illusion, a grotesque expression, which arises from viewing the eyes and mouth upside-down, is perceived only when the inverted features are presented in their usual orientation, that is, eyes above mouth. However, current information processing models of face recognition (e.g., Bruce \& Young, 1986) assume independent processing of identity and expression, an assumption based upon evidence from both experimental and neuropsychological studies. This evidence is more consistent with the hypothesis that the recognition of both facial identity and expression are disrupted by inversion because of the disruption of a common processing requirement, such as the processing of configural information, than with the hypothesis that analysis of facial identity is dependent upon analysis of expression.

Little or no direct evidence supports Yin's (1970) hypothesis. Phillips (1979) found that recognition of the internal features of a face, which are most prominently involved in the display of facial expression, was more disrupted by inversion than was recognition of the external features. This result is consistent with Yin's hypothesis, but is also open to other interpretations. Valentine and Bruce (1986) attempted to test Yin's expression hypothesis more directly by examining the effect of inversion upon the subsequent recognition of faces in subjects who had engaged in different encoding activities predicted to selectively enhance or reduce the encoding of expression. No support for Yin's hypothesis was found; the encoding activities did not affect recognition. However, this result could be due to the encoding activities' being ineffective in manipulating the encoding of expression. Parkin and Goodwin's (1983) finding that the recognition of faces whose expressions were changed was not affected by these encoding activities suggests that this may indeed be the case.

The majority of studies on the effect of orientation upon face recognition have examined recognition only for upright and inverted faces. However, at least two studies have measured performance at intermediate orientations between upright and inverted. Rock (1974) reported data on the recognition of famous faces in photographs presented upright but with the observer's head at $0^{\circ}, 45^{\circ}$, $90^{\circ}$, and $180^{\circ}$ angles relative to the vertical position. The data showed a monotonic decline in recognition performance as the observer's head was rotated away from the 
vertical, although no analysis of the function was reported. Cochran, Pick, and Pick (1983) used highly schematic profiles of faces in a mental rotation matching task. They found results that were highly dependent upon the nature of the task. Discriminating between identical patterns and their mirror image showed a linear relationship between reaction time (RT) and orientation. When the stimuli differed in the shape of individual features, a nonlinear function was obtained. Cochran et al.'s study proivdes data about the effects of task demands upon mental rotation tasks but, because of the highly artificial nature of the stimuli used, provides little insight into the processes used in face recognition.

In the experiments reported here, a mental rotation procedure was used to examine the effect of orientation upon face processing. The orientation of the stimuli was varied in $45^{\circ}$ steps between upright and inverted so that the nature of the function relating orientation and RT could be explored. Shepard and Metzler (1971) found that subjects' RT to decide whether two three-dimensional shapes were the same or different increased linearly with the difference in orientation of the two shapes to be compared. Our Experiment 1 also made use of a matching task, because this procedure has been used in many experiments in the mental rotation literature.

If faces can be processed as a perceptual Gestalt, it may be possible to mentally rotate a face as a whole. But Rock (1973) is explicit in postulating that inverted faces have to be rotated feature by feature. Although Carey and Diamond (1977) argue that the processing of inverted faces relies upon piecemeal information, they do not specify the manner in which inverted faces may be processed. However, Carey and Diamond's hypothesis also suggests that to rotate an inverted face, it may be necessary to rotate the features one at a time. If an inverted face can be rotated as a whole, it is difficult to imagine why configural information cannot be extracted. Rock's and Carey and Diamond's hypotheses suggest that it may be possible to rotate as a whole faces that deviate slightly from the upright position but that if faces are presented upside-down or nearly so, features may have to be rotated individually. Such a change in processing strategy might be revealed as a discontinuity in the relationship between $R T$ and orientation. Although it is extremely difficult to infer the nature of the underlying representation from mental rotation studies (e.g., Kosslyn, 1981; Pylyshyn, 1981; Shepard \& Cooper, 1982), Carey and Diamond's claim of a change in processing strategy would be strengthened if, for example, a discontinuity in the rotation function was found at an intermediate orientation between $0^{\circ}$ and $180^{\circ}$.

\section{EXPERIMENT 1}

The aim of Experiment 1 was to investigate whether a face recognition task would give a linear RT function similar to that found by Shepard and Metzler (1971) for block figures or show signs of a discontinuity with orien- tation. Earlier work (Valentine, 1986) had found that simultaneous presentation using faces as stimuli gave RTs that were very slow and variable. Therefore, sequential presentation was used in this experiment to obtain faster and less variable RTs. It should be noted that a linear relationship between RT and orientation has been found both in experiments in which three-dimensional figures were presented simultaneously (Shepard \& Metzler, 1971) and in experiments in which they were presented sequentially (Metzler \& Shepard, 1974).

An important consideration was to make the task as realistic as possible. Therefore, photographs of real faces served as the stimuli. This made the choices on the nature of the differences between the stimuli on different trials problematic. Shepard and Metzler used mirror images of the objects in the different trials. This difference is inappropriate for a face recognition task, because mirror reversals have a very small effect upon the recognition of faces (McKelvie, 1983). In this experiment, different trials were composed of photographs of two different people who were selected because their faces were similar. This means that the differences were less well controlled, but that the task had the advantage of being more realistic. As Cochran et al. (1983) demonstrated, the exact nature of the differences can have a powerful effect upon the results obtained. Therefore, this difference between the present experiment and previous work using a similar paradigm is important.

A second factor manipulated in the task was facial expression. This factor was included to explore Yin's (1970) hypothesis that disruption of facial expression processing is important in the impaired recognition of disoriented faces. Same trials consisted of either two identical pictures of faces or two pictures of the same face with different facial expressions, whereas different trials involved faces of two different people showing either the same or a different facial expression. Trials that involved a changed or unchanged facial expression were mixed in a random order, so the subject did not know for any trial whether there would be a change in expression. If the disproportionate effect of inversion upon face recognition is due to the difficulty of encoding the facial expression in an inverted face, two different predictions of the effect of changing expression on matching disoriented faces are possible.

First, it could be argued that since it is difficult to encode the expression of an inverted face, it would be more difficult to perceive that any differences between the upright and inverted faces might be due to a change of expression rather than to a different identity. Therefore, when pictures of the same person with different expressions are presented, it would be expected that the latency of "same" judgments might increase more rapidly with increasing displacement from the upright than when identical pictures were presented.

A prediction of the opposite interaction is also possible. It could be argued that if expression is difficult to perceive in an inverted face, subjects would not even no- 
tice that the expression was changed when the face was inverted. Therefore, for inverted faces, it should make no difference whether the expression is changed. This line of reasoning leads to the prediction that trials in which the expression is changed will show a smaller effect of inversion than will trials in which identical pictures are presented. This is because, when upright, identical pictures can be matched using a pictorial code (see Bruce, 1982). Therefore, upright identical pictures would be matched faster than faces involving an expression change, but when identical pictures are inverted, no effect of expression would be expected. Thus, Yin's hypothesis could lead to two opposite predictions, but could not account for the finding that face matching is equally impaired with inversion whether or not a change in expression is involved.

\section{Method}

Subjects. Fourteen 16-18-year-old high school students ( 3 male and 9 female) acted as subjects. They took part in a series of experiments for which they were paid $£ 3$. Data were discarded from 2 subjects who made errors on more than $15 \%$ of the trials; this left data available from 12 subjects.

Materials. Eight stimulus faces were used. These faces consisted of four visually similar pairs. The faces were selected to give pairs of faces that were similar in hair length and shade, were about the same age, and were of the same sex. Two pairs were male, and two pairs were female. The faces (two expressions of each face, smiling and unsmiling) were copied individually onto monochrome $35 \mathrm{~mm}$ slides. Copies were made at five orientations with respect to upright (at $0^{\circ}, 45^{\circ}, 90^{\circ}, 135^{\circ}$, and $180^{\circ}$ ).

Apparatus. Slides were presented using a three-field projection tachistoscope. Three Kodak Carousel projectors projected slides for each field onto the same area of a blank wall. The subject sat to the left of the projectors. Each face subtended approximately $5^{\circ}$ of visual angle. The subject indicated his/her response using push buttons. A Rockwell AIM 65 microprocessor recorded the subject's response and $\mathrm{RT}$ and controlled the projectors.

Design. The first face of each pair was always presented upright. After a short pause, the second face of the pair was presented. This face could be in any one of five orientations in $45^{\circ}$ steps from upright to $180^{\circ}$ of rotation in a clockwise direction. In half the trials, the two faces were of the same person, (same trials); in half, they were of two visually similar people (different trials). In different trials, each face was always paired with the visually similar face. Thus, different trials for Item 1 consisted of Item 2 followed by Item 1; different trials for Item 2 consisted of Item 1 followed by Item 2. A second factor was facial expression. In half the trials, both faces of each pair had the same facial expression, either smiling or unsmiling; in half the trials, the expression was different. Therefore, the design involved two within-subjects factors for both same and different trials: expression change and orientation change. There were 160 trials, 8 (items) $\times 5$ (orientation) $\times 2$ (expression change) $\times 2$ (same/different).

Procedure. The subjects were tested individually. There was a warning tone $500 \mathrm{msec}$ before the start of each trial. The first face was presented for $1,500 \mathrm{msec}$; following a 250 -msec blank field, the second face was presented for $2,000 \mathrm{msec}$. The start of the next trial was $3 \mathrm{sec}$ after the subject had indicated his/her response by using push buttons. The subjects were told that the second face of each pair could be disoriented and that on some trials the facial expression would be changed. They were told that in different trials visually similar faces would be paired together and that the same four pairs would be used for different trials throughout the experiment. The subjects were told to respond as quickly as possible without making unnecessary errors. Half of the subjects pressed the same button with the preferred hand; the other subjects pressed the same button using the nonpreferred hand. Each subject was given 20 practice trials, in which the faces were different from those used in the experiment. The 160 trials were presented in two blocks of 80 trials. The slide magazines were changed between blocks. A random order was constructed with the following constraints: (1) there were no more than three consecutive same or different trials, (2) there were no more than three consecutive trials with the same or different expression, and (3) no consecutive trials involved pictures of the same person or the same orientation of the second face. Four different orders were made by reversing the sequence of presentation of the slide magazines and by changing the position of the two halves of the list within each magazine. Three subjects saw the slides in each order.

\section{Results}

Separate analyses of RTs in correct same and different trials were carried out. These data are plotted in Figures 1 and 2 . The latencies of correct decisions were converted into RT in milliseconds prior to being subjected to analysis of variance (ANOVAs). A two factor within-subjects ANOVA of same latencies revealed a main effect of orientation $[F(4,44)=21.27, M S e=0.0080, p<.0001]$ and expression change $[F(1,11)=59.87, M S e=0.0118$, $p<.0001]$. RTs increased with increases in rotation from upright and were slower when the expression was different than when it was the same (see Figure 1). When upright identical picture matches were made an average of $122 \mathrm{msec}$ faster than matches involving a changed expression ( $M \mathrm{~s}=642$ and $764 \mathrm{msec}$, respectively). When the second face was inverted, this difference was $204 \mathrm{msec}(M \mathrm{~s}=792$ and $996 \mathrm{msec}$, respectively).

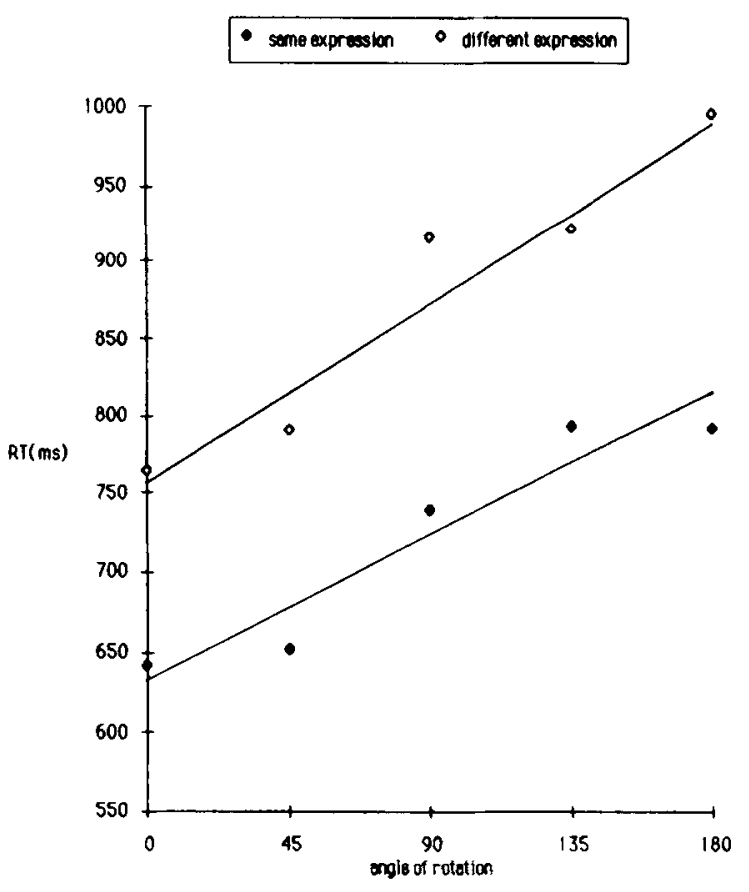

Figure 1. Mean reaction times (RTs, in msex) of "same" responses from Experiment 1, plotted as a function of angle of rotation in degrees and expression. 


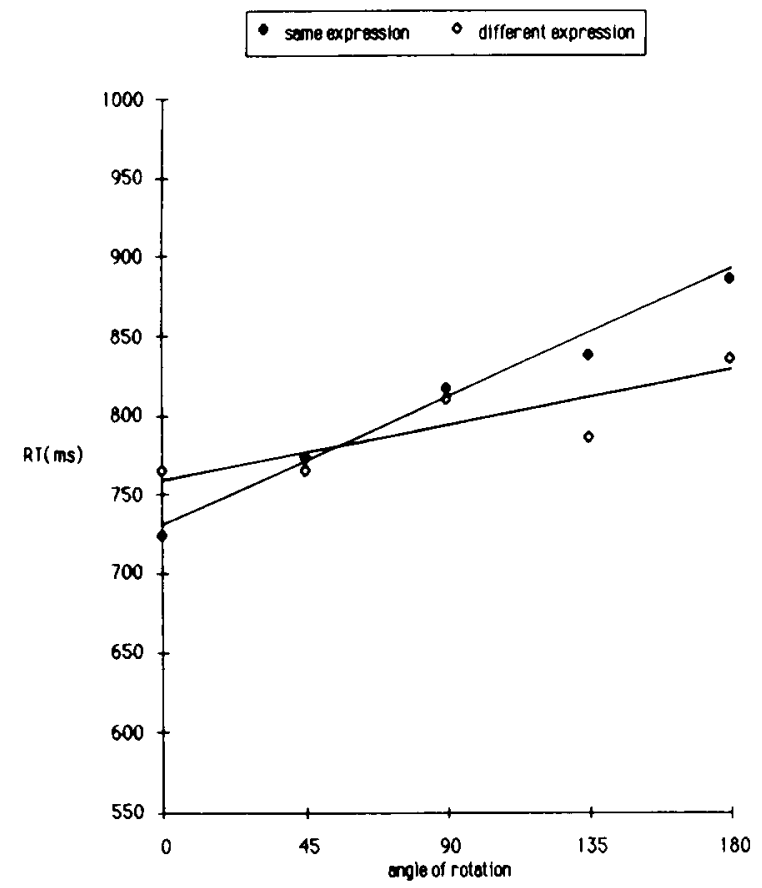

Figure 2. Mean reaction times (RTs, in msec) of "different" responses from Experiment 1, plotted as a function of angle of rotation in degrees and expression.

Although there was a trend for this difference to increase with orientation, the interaction between expression and orientation did not even approach statistical significance $(F=1.0)$. A similar analysis of different latencies revealed a main effect of orientation $[F(4,44)=13.97, M S e$ $=0.0034, p<.0001]$ but no main effect of expression change $(F<1)$. The trend for the interaction was in the opposite direction to the trend observed for the same trials but was not statistically significant $[F(4,44)=1.91, M S e$ $=0.0045, p>.1$.

A combined analysis of "same" and "different" response latencies was also carried out. There were main effects of orientation $[F(4,44)=38.77, M S e=0.0051$, $p<.0001]$ and expression change $[F(1,11)=27.15$, $M S e=0.0106, p<.001]$, and there was an interaction between trial type and expression change $[F(1,11)=$ $30.11, M S e=0.0142, p<.001]$. The interaction was due to changed expression affecting the latency of same trials but not the latency of different trials as found in the separate analyses. There was also an interaction between trial type and orientation $[F(4,44)=3.48, M S e=0.0064$, $p<.02]$. Orientation had a larger effect upon the response latencies of same trials than upon those of different trials. Although no other effects were statistically significant, the three-way interaction approached significance $[F(4,44)=2.35, M S \mathrm{e}=0.0050, p<.07]$, reflecting the opposite trends for the interaction terms found in the separate analyses of same and different trials.

Linear regression analyses were carried out on the response latencies of correct same and different trials. Regression lines are shown in Figures 1 and 2. The linear component was significant at the $p<.01$ level in the analysis of different trials involving different expressions and at the $p<.001$ level in all other analyses. In none of these analyses was the nonlinear component significant.

Errors were made on $8.2 \%$ of the trials; the distribution of errors across orientation is shown in Table 1. Because the error rates in some conditions were reasonably high, an analysis of error scores was also carried out. However, because of the small number of items making up data for each individual cell and the low error rates in some conditions, there was a high proportion of zero cells. Therefore, raw error scores were subjected to a $\sin ^{-1} \sqrt{(x+3 / 8) /(n+3 / 4)}$ transformation before an ANOVA was carried out (Johnson \& Leone, 1964).

An ANOVA of transformed error scores for same trials revealed a main effect of expression change $[F(1,11)=$ $44.45, M S e=0.031, p<.001]$. More errors were made on trials in which the facial expression was changed than on trials in which both faces displayed the same expression. There was also a main effect of orientation $[F(4,44)$ $=9.08, M S e=0.010, p<.001]$. The interaction between expression and orientation was not significant $[F(4,44)=1.83, M S e=0.023, p>.1]$. The effect of orientation was explored further using Newman-Keuls tests with a confidence level of .05 . None of the pairwise comparisons between orientations differed in trials involving an unchanged facial expression. In trials in which the facial expression was changed, the error rate to stimuli presented at $135^{\circ}$ to the upright was greater than the error rate to stimuli presented at all other orientations. More errors were made to inverted faces $\left(180^{\circ}\right)$ than to faces presented at upright $\left(0^{\circ}\right)$ or at $45^{\circ}$. The analysis of error scores is consistent with the analysis of RTs in showing no interaction between the effects of orientation and expression change. In addition, the error score analysis does not provide any evidence that a speed/accuracy tradeoff substantially influenced the results.

A similar analysis of transformed error scores in different trials showed no significant effects (all $F$ ratios $<1$ ).

\section{Discussion}

This experiment found a linear relationship between the difference in orientation of two faces and RT in a matching task. This relationship applies to both same and different judgments in trials involving either an unchanged or changed expression. Thus, no evidence that matching two upright faces involves a qualitatively different process from matching one upright and one inverted face has been

Table 1

Mean Percentage of Errors as a Function of Angle of Rotation, Type of Trial, and Expression, from Experiment 1

\begin{tabular}{llrrrrr}
\hline & & \multicolumn{5}{c}{ Angle of Rotation } \\
\cline { 3 - 7 } Expression & Trial Type & \multicolumn{1}{c}{$0^{\circ}$} & \multicolumn{1}{c}{$45^{\circ}$} & $9^{\circ}$ & $135^{\circ}$ & $180^{\circ}$ \\
\hline \multirow{2}{*}{ Unchanged } & Same & 1.0 & 2.0 & 3.1 & 4.1 & 2.0 \\
& Different & 2.0 & 10.4 & 5.2 & 5.2 & 7.2 \\
\multirow{2}{*}{ Changed } & Same & 16.7 & 10.4 & 16.7 & 33.3 & 21.9 \\
& Different & 5.2 & 5.2 & 4.1 & 7.2 & 6.2 \\
\hline
\end{tabular}


found. The linear relationship found in this experiment is consistent with the literature on mental rotation of threedimensional block drawings (e.g., Metzler \& Shepard, 1974; Shepard \& Metzler, 1971) and two-dimensional random polygons (e.g., Cooper, 1975).

Although this experiment found no evidence that a switch in processing strategy for matching faces takes place as disorientation is increased, such a possibility cannot be totally excluded for two reasons. First, it is possible that there is a change in processing strategy that is not revealed as a discontinuity in the rotation function. Second, RT was measured at five orientations and, although a linear function provided the best fit to the data, the points do show some deviation from a straight line. The possibility that the function does deviate slightly from being linear cannot be excluded. This point is discussed further in the General Discussion.

The analysis of the same trials showed that the latency to decide whether two faces are the same is increased if the expression is different in the two pictures. This effect is consistent with a match being made at the level of a pictorial code when an identical picture match can be made (cf. Bruce, 1982). This advantage for an identical picture persists even when the second picture is presented upside-down. The absence of an expression $x$ orientation interaction in same trials implies that difficulty in extracting information on expressions is not a major factor in the difficulty in matching inverted faces.

Although there was a main effect of expression in the analysis of same trials, no main effect of expression was found in the analysis of different trials. This result implies that facial expression was not an important factor in deciding that the two pictures were of different people. However, there was a trend, which failed to reach statistical significance, for an interaction between expression and orientation in different trials. Examination of Figure 2 reveals that this trend reflects a crossover interaction, perhaps suggesting that when a face is disoriented, there is a tendency for any difference in the stimuli to facilitate a different response.

In summary, Experiment 1 used a matching task to examine the role of both Carey and Diamond's (1977) piecemeal-configural information distinction and Yin's (1970) expression hypothesis in accounting for the effect of inversion upon face recognition. No support for either hypothesis was found. One possible objection to Experiment 1 is that a matching task was used although both hypotheses concern the recognition of faces. Therefore, one aim of Experiment 2 was to establish whether a linear function between orientation and RT would be obtained in a recognition task. The second aim was to examine Yin's (1970) hypothesis further. Experiment 1 and Valentine and Bruce's (1986) Experiment 3, which made use of encoding activities, both failed to support Yin's expression hypothesis. However, rejecting Yin's hypothesis on the basis of these experiments relies upon accepting negative results. Therefore, this evidence does not amount to a total refutation of the hypothesis. A further aim of Ex- periment 2 was to establish whether disruption of expression analysis could account for the disproportionate effect of inversion upon face recognition.

\section{EXPERIMENT 2}

If difficulty in the recognition of expression can account for the disproportionate effect of inversion upon recognizing faces, judgments of expression must be at least as disrupted by inversion as are judgments of identity. If, however, recognition of faces is more disrupted by inversion than is recognition of expression, the difficulty in recognizing inverted faces cannot be accounted for by the role of expression. The effect of inversion upon different types of judgments involving faces was compared in Experiment 2. Four different tasks were used. The first involved distinguishing between faces and common objects (object decision task). A second involved distinguishing between faces and jumbled faces (jumbled-face decision task). The final two tasks involved deciding whether each face was smiling (expression decision task) and whether each face was familiar (familiarity decision task). These different tasks were designed to require processing different levels within the face recognition system. The object decision task is an extremely simple discrimination, and it was expected that this task would be affected little, if at all, by inversion. The jumbled-face decision task requires more processing, because it involves a decision concerning the layout of the internal features of the face. However, it does not involve any recognition of individual identity or of facial expression. The expression decision task and the familiarity decision task were designed to require processing of these two different aspects of faces. The critical question was whether the familiarity decision task would be more impaired by inversion of the stimulus than either the expression decision task or the jumbled-face decision task. If so, this would demonstrate that the disproportionate effect of inversion results from the assignment of individual identity to a face rather than from the recognition of either facial expression or the facial pattern.

\section{Method}

Subjects. Twenty undergraduate students ( 9 male, 11 female) were paid $£ 2$ to take part in two experiments. None were from the Psychology Department.

Materials. For each task, five positive and five negative items, which were repeated at each of five orientations, were used. For the object decision task, five faces of unfamiliar males were used in the positive trials and photographs of five common objects (a house, a cup, a robin, a clock, and a train) were used in the negative trials.

For the jumbled-face decision task, five different unfamiliar male faces were used for both the positive and the negative trials. The positive trials consisted of ordinary photographs of faces, but they had a rectangle drawn around the eyes, nose, and mouth region. Horizontal lines separated this rectangle into three smaller rectangles each containing one of the features (eyes, nose, or mouth). The jumbled faces were made by cutting out these rectangles and reassembling the face with the features in a wrong order. Several 

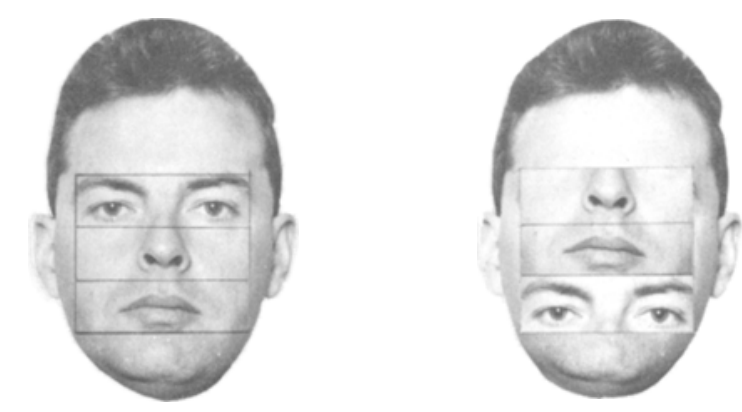

Figure 3. An example of an intact and a jumbled face used in the jumbled-face decision task of Experiment 2.

different orders of features were used. The lines were drawn on the faces for the positive trials so that the faces could not be categorized by the presence of lines alone. An example of an intact and a jumbled face is shown in Figure 3.

For the expression decision task, five faces of unfamiliar females who were all smiling broadly were used in the positive trials and pictures of the same people posing with different expressions (neutral, disgust, anger, and surprise) were used in the negative trials. These different expressions were used so that the decision could not be based on the presence or absence of a single feature such as visible teeth or an open mouth. In this task, different pictures were used for four of the five orientations to make up the negative trials. Since there were four expressions and five orientations, one expression was repeated for each actress. The repeated expression and the orientation involved was different for each actress.

In the familiarity decision task, five pictures of famous males ${ }^{1}$ (positive items) and five pictures of unfamiliar males were used.

Pictures of all items were cut out, to exclude all background, and copied onto monochrome $35 \mathrm{~mm}$ slides against a plain white background. Except for the negative expression trials, in which different expressions appeared at different orientations, all items were copied at all five orientations $\left(0^{\circ}, 45^{\circ}, 90^{\circ}, 135^{\circ}\right.$, and $\left.180^{\circ}\right)$. No item appeared in more than one task.

Apparatus. Slides were projected from a Kodak Carousel projector onto a blank wall. The pictures subtended a visual angle of approximately $8^{\circ}$. The subject sat to the left of the projector, which was controlled by a Rockwell AIM 65 microprocessor. The microprocessor recorded the subjects' RTs and decisions. The subjects indicated their decisions by pushing two push buttons.

Design. A within-subjects design was used. The subjects carried out all four tasks. The order in which the tasks were presented was counterbalanced using a Latin-square design producing four different task orders. Five subjects saw the tasks in each order. Four different slide orders within each task were used. Two random orders were generated using the following constraints: (1) there were no more than three consecutive positive or negative trials, (2) the same item was not repeated on consecutive trials, and (3) the same orientation was not repeated on consecutive trials. The two remaining orders were generated by reversing the order in which the two halves of the lists were presented. The assignment of slide order to task was also made on the basis of the Latin-square procedure used. The dependent variable was the subject's response latency. The design consisted of two within-subjects factors (task and orientation) and one between-subjects factor (task order), although task order was confounded with slide order.

Procedure. The nature of the first task was explained. The decisions were described as yes or no decisions. The questions were, "Is it a face?"' (as opposed to an object or a jumbled face), "Is the face smiling?", and "Is the face familiar?" The subjects were shown prints of examples of some positive and negative practice trials. They were informed that RT was being measured and were asked to respond as quickly but still as accurately as possible. Each task of 50 trials was preceded by 5 practice trials of items not included in the experimental trials. A blank trial was inserted after the practice trials so that the experimental sequence did not start until the subject had pressed a button to indicate that he/she was ready. A warning tone preceded each trial by $500 \mathrm{msec}$. The slides were presented for $1,500 \mathrm{msec}$ with an intertrial interval of $2,000 \mathrm{msec}$. (This interval was timed from the subject's response if the RT was greater than $1,500 \mathrm{msec}$.)

The procedure for the familiarity decision task was slightly different. Previous work had indicated that subjects perform near chance when required to identify inverted famous faces in a familiarity decision task. Therefore, the subject initially was shown prints of the two famous faces that had been used in the practice trials. After it was established that the subject recognized these faces, he/she was told that all other faces would be of unfamiliar people. The slide sequence stopped after the practice trials, as described above. The subject was then shown prints of the five famous faces that would be seen in the experimental trials. After it was again established that the subject recognized these faces, he/she was informed that all other faces presented in the experimental trials would be of unfamiliar faces. When the subject pressed a button to indicate that he/she was ready, the experimental trials were presented.

\section{Results}

Latencies from positive and negative trials were analyzed separately. Positive responses will be discussed first.

Positive (yes) responses. Errors were made on 1.5\% of the trials. The distribution of errors was as follows: object decision task, $0.4 \%$; jumbled-face decision task, $2 \%$; expression decision task, $1.4 \%$; familiarity decision task, $2.4 \%$. The distribution of errors across orientation is given in Table 2 . Errors were not analyzed further because over $90 \%$ of the cells in an analysis would be zero cells.

The group mean RTs of correct positive judgments are plotted in Figure 4. These data were converted into RT in seconds and subjected to a reciprocal transform before being entered into a $4 \times 4 \times 5$ split-plot ANOVA. The transformation was done to remove the relationship between group means and standard deviation in the data (Kirk, 1968). This relationship is found because of the large range in group mean RTs. The order in which the tasks were carried out was a between-subjects factor; the different tasks and orientation were within-subjects factors. There was a significant main effect of task $[F(3,48)=$ 101.07, $M S \mathrm{e}=0.0868, p<.0001]$. Examination of Figure 4 shows that this effect was mainly due to the fact that RTs were much faster in the object decision task than in the other tasks. There was also a main effect of orien-

Table 2

Mean Percentage of Errors as a Function of Angle of Rotation and Task, in Positive Trials from Experiment 2

\begin{tabular}{lllccc}
\hline & \multicolumn{5}{c}{ Angle of Rotation } \\
\cline { 2 - 6 } \multicolumn{1}{c}{ Task } & $0^{\circ}$ & $45^{\circ}$ & $90^{\circ}$ & $135^{\circ}$ & $180^{\circ}$ \\
\hline Object & 1.0 & 1.0 & 0 & 0 & 0 \\
Jumbled Face & 3.0 & 3.0 & 0 & 2.0 & 2.0 \\
Expression & 1.0 & 3.0 & 1.0 & 0 & 2.0 \\
Familiarity & 1.0 & 1.0 & 2.0 & 2.0 & 6.0 \\
\hline
\end{tabular}




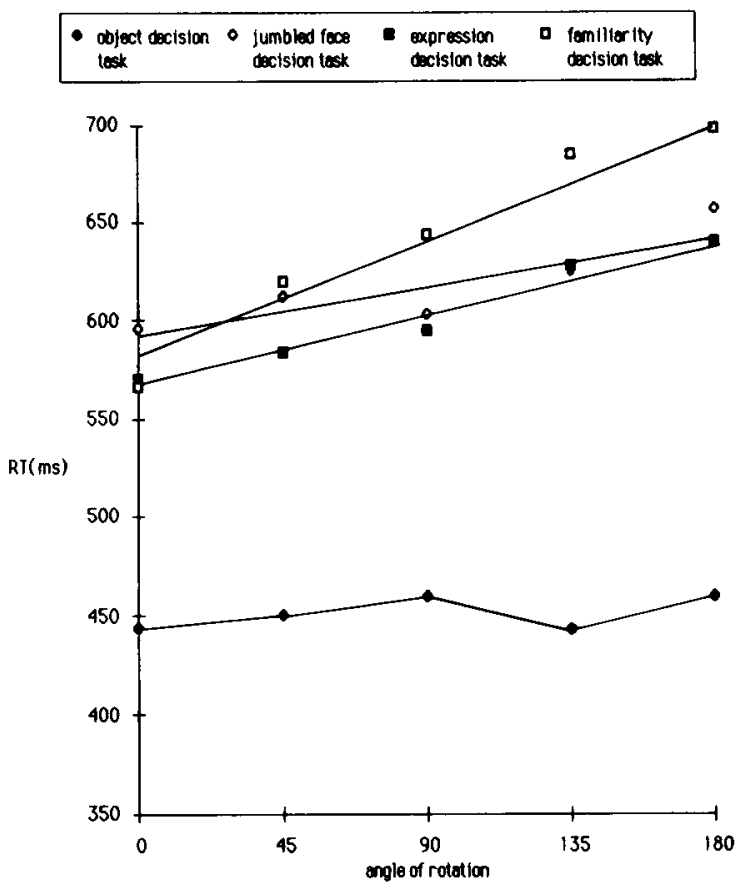

Figure 4. Mean reaction times (RTs, in msec) of "yes" responses from Experiment 2, plotted as a function of angle of rotation in degrees and task.

tation $[F(4,64)=18.95, M S e=0.0208, p<.001] . \mathrm{RTs}$ to disoriented items were slower than RTs to upright items.

The predicted task $\times$ orientation interaction was significant $[F(12,192)=3.86, M S e=0.0155, p<.001]$. Analysis of simple main effects revealed that there was a main effect of orientation for the familiarity decision task $[F(4,64)=16.89, p<.0001]$, the expression decision task $[F(4,64)=5.59, p<.001]$, and the jumbledface decision task $[F(4,64)=3.98, p<.01]$, but not for the object decision task $[F(4,64)=1.16, p=.35]$. The prediction was that the effect of orientation should be greater for the familiarity decision task than for either the expression or jumbled-face decision tasks. The simple main effects reported above do not enable this conclusion to be drawn. Therefore, treatment-contrast interaction analyses were carried out for the comparisons of interest (Kirk, 1982). As two comparisons were being made, the significance level accepted was adjusted to $.025(.05 / 2)$. These comparisons revealed that the effect of orientation was greater for the familiarity decision task than for either the expression decision task $[F(4,192)=3.42, p<.02]$ or the jumbled-face decision task $[F(4,192)=4.49$, $p<.002]$.

The ANOVA also gave a significant three-way interaction between order $\times$ task $\times$ orientation $[F(36,192)=$ $1.49, M S \mathrm{e}=0.0155, p<.05]$. This reflected a learning effect, such that the effect of orientation was greater for the initial tasks. No other effects were significant (all $p$ values $>.15$ ).
Linear regression analyses of the RT data were carried out. A significant linear component was found for all tasks except the object decision task (at the $p<.001$ level in all cases). Regression lines are plotted in Figure $\mathbf{4}$ for data that gave a significant linear component. In none of the analyses was the nonlinear component significant.

Negative (no) responses. Errors were made on $1.2 \%$ of the trials. The distribution of errors was as follows: object decision task, $0.8 \%$; jumbled-face decision task, $2.8 \%$; expression decision task, $3.2 \%$; familiarity decision task, $2.0 \%$. The distribution of errors across orientation is given in Table 3. Errors were not analyzed further because over $90 \%$ of cells in an analysis would be zero cells.

The mean latencies from correct negative decisions are plotted in Figure 5. A three-way ANOVA was carried out on the reciprocal-transformed RT data in seconds, as was done for the analysis of positive trials. All of the significant effects in the ANOVA carried out on the positive trials were also significant in the analysis of the negative trials. There was a significant main effect of task $[F(3,48)=242.43, M S \mathrm{~S}=0.0396, p<.0001]$ and orientation $[F(4,64)=31.25, M S e=0.0143$, $p<.0001]$. The interaction between task and orientation $F(12,192)=4.24, M S e=0.0142, p<.0001]$ and the order $\times$ task $\times$ orientation $[F(36,192)=2.09, M S e$ $=0.0142, p<.001]$ interaction were significant. The interaction between order and task, which was not significant in the analysis of the positive trials, did achieve statistical significance in the analysis of the negative trials $[F(9,48)=2.33, M S e=0.0396, p<.05]$. This effect does not appear to be of any theoretical interest and was not analyzed further.

Analysis of the simple main effects of orientation revealed a significant effect for all tasks [familiarity decision task, $F(4,64)=18.45, p<.001$; jumbled-face decision task, $F(4,64)=16.15, p<.001$; expression decision task, $F(4,64)=5.04, p<.005$; object decision task, $F(4,64)=4.35, p<.005$ ]. Analysis of treatmentcontrast interactions revealed that the effect of orientation was greater for the familiarity decision task than for the expression decision task $[F(4,192)=5.80$, $p<.001]$, but there was no difference in the effect of orientation upon the familiarity decision task and the jumbled-face decision task $(F<1)$.

Linear regression analyses of RTs were carried out. Regression lines are plotted in Figure 5 for data that gave

Table 3

Mean Percentage of Errors as a Function of Angle of Rotation and Task, in Negative Trials from Experiment 2

\begin{tabular}{lccccc}
\hline & \multicolumn{5}{c}{ Angle of Rotation } \\
\cline { 2 - 6 } \multicolumn{1}{c}{ Task } & $0^{\circ}$ & $45^{\circ}$ & $90^{\circ}$ & $135^{\circ}$ & $190^{\circ}$ \\
\hline Object & 0 & 2.0 & 1.0 & 1.0 & 0 \\
Jumbled Face & 0 & 5.0 & 2.0 & 5.0 & 5.0 \\
Expression & 5.0 & 6.0 & 2.0 & 2.0 & 1.0 \\
Familiarity & 1.0 & 0 & 1.0 & 3.0 & 5.0 \\
\hline
\end{tabular}




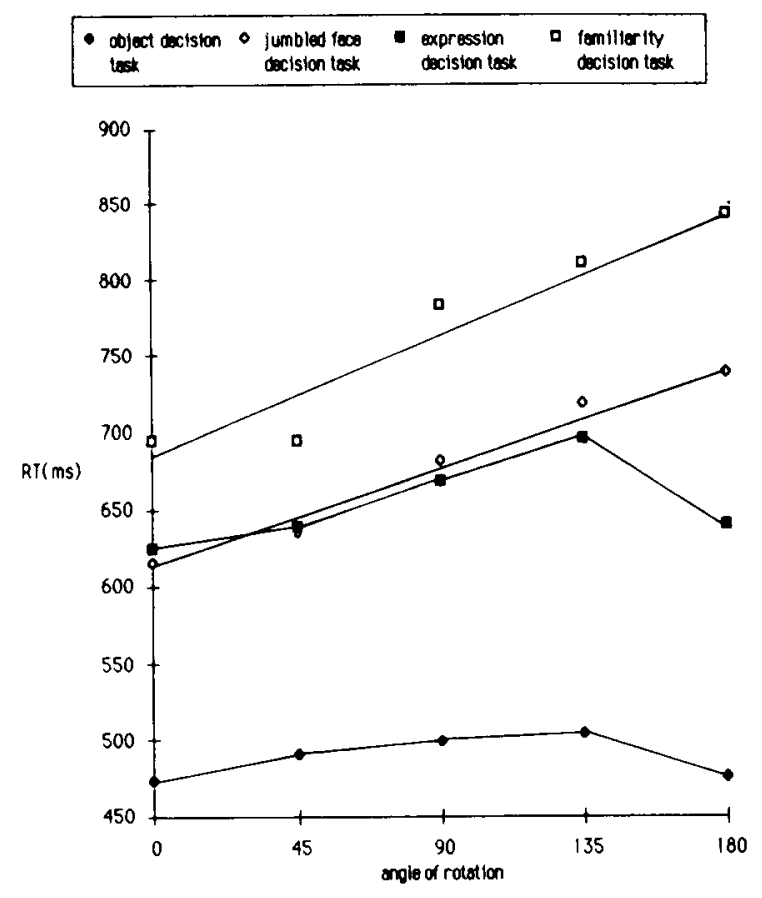

Figure 5. Mean reaction times (RTs, in msec) of "no" responses from Experiment 2, plotted as a function of angle of rotation in degrees and task.

a significant linear component $(p<.001$ in both cases) and a nonsignificant nonlinear component. Both the expression decision task and the object decision task gave significant nonlinear components. Further trend analysis revealed a significant quadratic component $(p<.001)$ and a nonsignificant higher order component in both cases.

\section{Discussion}

This experiment has found a linear relationship between the time taken to accept a famous face as familiar and the orientation of the face. This result provides no evidence to support the hypothesis that inverted faces are processed in a qualitatively different way from upright faces. This finding, taken together with the results of Experiment 1, indicates a linear relationship for both a matching and a recognition task.

In the analysis of the positive responses, it was found that there was a greater effect of inversion upon the familiarity decision task than upon either the expression decision task or the jumbled-face decision task. This result is inconsistent with the view that expression analysis is a sufficient factor to account for the disproportionate effect of inversion upon face recognition. The object decision task produced very fast RTs and showed no main effect of inversion. This result suggests that task difficulty may interact with orientation. However, the greater effect of orientation for the familiarity decision task cannot be attributed to task difficulty, because the upright trials of the familiarity decision task gave faster RTs than did the upright trials of the jumbled-face decision task and the expression decision task.
Generally, the pattern of latencies for the negative trials is similar to the pattern of latencies for the positive trials, although less consistent. The object decision task gave much faster RTs than did the other tasks. The effect of orientation upon the object decision task latencies was significant for the negative trials, but the linear component was not statistically significant for either positive or negative trials. Orientation had a greater effect upon the familiarity decision task than on the expression decision task, but there was no difference in the effect of orientation upon the familiarity decision task and the jumbledface decision task. However, there are important differences in the nature of the negative trials between the different tasks. The jumbled-face decision task and the expresion decision task used faces of the same actors/actresses in the positive and negative trials whereas the task demands of the object decision task and the familiarity decision task obviously require different items to be used. In addition, different items were used at different orientations in the negative trials of the expression decision task.

In conclusion, this experiment has found no evidence that inverted faces are processed in a qualitatively different way from upright faces. No sign of a switch in strategy from configural to piecemeal processing is found when the $\mathrm{RT}$ to recognize a familiar face is plotted against orientation. In addition, it was found that disorientation has a greater effect upon a task requiring a familiarity judgment than on a task requiring an expression judgment for both positive and negative responses. This result provides positive evidence that disruption of the analysis of expression is insufficient to account for the disproportionate effect of inversion upon the recognition of faces. This result, taken together with the negative results of Valentine and Bruce (1986, Experiment 3) and the present Experiment 1, establishes that Yin's hypothesis can be rejected. Some factor other than expression must be sought to account for the disproportionate effect of inversion.

\section{GENERAL DISCUSSION}

Experiment 1 found that RT in a sequential facematching task increases linearly as the orientation of the second face deviates further from upright. Experiment 2 also found a linear relationship in a face recognition task. These results are broadly consistent with earlier work on the effect of orientation upon matching and recognizing other stimulus materials.

The RT functions obtained do not show a sharp discontinuity that might be expected if disorientation causes a switch in processing strategy as proposed by Carey and Diamond (1977). Similarly, Flin (1985) found no evidence of a switch in processing strategy in a developmental study of the effect of inversion upon face recognition. Therefore, these results do not show any support for Carey and Diamond's hypothesis. However, a switch in processing strategy cannot be totally excluded by the results of the present study. It is possible that inversion does cause a 
switch from processing configural to piecemeal information, which cannot be detected in the mental rotation function. Alternatively, the function may deviate ony slightly from a linear function. For example, if the function is slightly S-shaped, this should be revealed as a cubic component. However, it is possible that the procedure used is too insensitive to detect this component. Further work in which RT is measured at more orientations would be needed to explore this possibility.

Both experiments examined the effect of facial expression in processing disoriented faces. Experiment 1 found no interaction between the effects of expression and orientation in a face matching task in same trials although there was a nonsignificant trend for an interaction in the RT of different trials. Experiment 2 found that the RT of judgments of facial identity increased more rapidly with orientation than the RT of judgments of facial expression. These results are inconsistent with the view that facial expression is a major factor in accounting for the influence of orientation upon recognition of facial identity. In summary, orientation has been found to have a strong influence upon the time taken to match and recognize faces, but no evidence has been found to support the hypothesis that inverted faces are processed in a qualitatively different manner from upright faces.

If there is no switch in processing strategy, how can the disproportionate effect of inversion be explained? $\mathrm{Di}$ amond and Carey (1986) proposed a critical role for second-order relational information in their account of the effect of expertise in discriminating within a stimulus class. They argued that recognition of upright faces would depend heavily upon encoding features that incorporate such information. However, second-order relational information cannot be extracted from inverted faces. Therefore, the recognition of inverted faces must rely upon different features, ones that can be described in terms of component information alone. Thus, Diamond and Carey argued that the features used to recognize inverted faces had to be different from those used to recognize upright faces.

However, an account of the disproportionate effect of inversion upon face recognition in terms of second-order relational information does not necessarily demand that a qualitative difference in the processing of upright and inverted faces is postulated. It is sufficient to argue that recognition of faces is especially dependent upon the encoding of second-order relational information (because they form a highly homogeneous class of which many exemplars are experienced) and that inversion impairs the encoding of this information but does not render it impossible to encode. The essential difference between this interpretation and Diamond and Carey's is that this view suggests that the effect of orientation can be seen as a quantitative effect that impairs face recognition but does not necessarily cause a qualitative change in the features used to recognize faces.

To summarize, although there is evidence that the disproportionate effect of inversion upon face recognition does appear to be due to an impairment in the processing of configural (or second-order relational) information, this study has found no evidence of a switch from the processing of configural information in upright faces to a piecemeal processing of individual features in inverted faces (cf. Carey \& Diamond, 1977; Rock, 1973). The results of the present study are more consistent with the view that recognition of upright and inverted faces depends upon the processing of the same featrues (cf. Flin, 1985; Goldstein \& Chance, 1980). There was no evidence to support the hypothesis that impaired encoding of second-order relational information disrupts the interpretation of expression in upside-down faces, which in turn disrupts recognition of facial identity (cf. Yin, 1970).

In their study of the mental rotation of three-dimensional block figures, Shepard and Metzler (1971) found linear relationships between $\mathrm{RT}$ and angle of rotation both for trials in which a rotation in depth was required and for trials in which a rotation in the picture plane was required. Rotations in depth were carried out no more slowly than rotations in the picture plane, suggesting that the RT for mental rotation was unaffected by differences in the twodimensional projections of the three-dimensional block figures. Our Experiment 1 found a linear function between RT and angle of rotation for a different class of threedimensional objects (faces) rotated in the picture plane. Comparison with Shepard and Metzler's results would suggest that depth rotations of faces (i.e., matching faces that differ in pose) would also show a linear relationship between RT and angle of rotation.

Bruce, Valentine, and Baddeley (1987) were primarily concerned with testing the hypothesis that there might be a canonical role for the three-quarters view of a face, but used a method analogous to that used in our Experiment 1 to examine rotations in depth. In Bruce et al.'s case, however, the results were very different from those of Shepard and Metzler (1971). The rotation functions were dependent upon the specific views involved and appeared to be determined by the visual similarity of the different views. For example, a match between full-face and profile views $\left(90^{\circ}\right.$ rotation) took longer than a match involving two full-face views, but a match involving opposite three-quarter views $\left(90^{\circ}\right.$ rotation) was matched faster than a profile and a three-quarter view $\left(45^{\circ}\right.$ rotation). Differences between familiar and unfamiliar faces were also found. For unfamiliar faces, a match involving two left three-quarter views were matched faster than two fullface views. This difference was not found for familiar faces. These results show that some factors other than angle of rotation are needed to account for the functions observed in the depth rotation of faces.

\section{REFERENCES}

Bruce, V. (1982). Changing faces: Visual and non-visual coding processes in face recognition. British Journal of Psychology, 73, 105-116.

Bruce, V., Valentine, T., \& Baddeley, A. (1987). The basis of the 
$3 / 4$ view advantage in face recognition. Applied Cognitive Psychology, 1, 109-120.

BRUCE, V.. \& Young, A. (1986). Understanding face recognition. British Journal of Psychology, 77, 305-327.

CAREY, S. . Diamond, R. (1977). From piecemeal to configurational representation of faces. Science, 195, 312-314.

Cochran, E. L., Pick, A. D., \& Pick, H. L. (1983). Task-specific strategies of mental "rotation" of facial representations. Memory \& Cognition, 11, 41-48.

COOPER, L. A. (1975). Mental rotation of random two-dimensional shapes. Cognitive Psychology, 7, $20-43$.

Diamond, R., \& Carey, S. (1986). Why faces are and are not special: An effect of expertise. Journal of Experimental Psychology: General, 115, 107-117

Fun, R. H. (1985). Development of face recognition: An encoding switch? British Journal of Psychology, 76, 123-134.

Goldstein, A. G., Chance, J. E. (1980). Memory for faces and schema theory. Journal of Psychology, 105, 47-59.

JoHNSON, N. L., LEONE, F. C. (1964). Statistics and experimental design: In engineering and the physical sciences. New York: Wiley.

KIRK, R. E. (1968). Experimental design: Procedures of the behavioral sciences (Ist ed.). Belmont, CA: Brooks/Cole.

KIRK, R. E. (1982). Experimental design: Procedures for the behavioral sciences (2nd ed.). Belmont, CA: Brooks/Cole.

KossLYN, S. M. (1981). The medium and the message in mental imagery: A theory. Psychological Review, 88, 46-66.

Maruyama, K., Endo, M. (1983). The effect of face orientation upon apparent direction of gaze. Tohoku Psychologica Folia, 42, 126-138

Maruyama, K., Endo, M. (1984). Illusory face dislocation effect and configural integration in the inverted face. Tohoku Psychologica Folia, 43, 150-160.

MCKeLVIE, S. J. (1983). Effects of lateral reversal on recognition memory for photographs of faces. British Journal of Psychology, 74, 391-407.

Metzler, J., ShePard, R. N. (1974). Transformational studies of the internal representation of three-dimensional objects. In R. L. Solso (Ed.), Theories of cognitive psychology: The Loyola symposium (pp. 147-201). Potomac, MD: Erlbaum.

PARKIN, A. J., Goodwin, E. (1983). The influence of different processing strategies on the recognition of transformed and untransformed faces. Canadian Journal of Psychology, 37, 272-277.

Parks, T. E., Coss, R. G., \& Coss, C. S. (1985). Thatcher and the Cheshire cat: Context and the processing of facial features. Perception, 14, 747-754.
PhILlips, R. J. (1979). Some exploratory experiments on memory for photographs of faces. Acta Psychologica, 43, 39-56.

Pylyshyn, Z. W. (1981). The imagery debate: Analogue media versus tacit knowledge. Psychological Review, 88, 16-45.

Rock, I. (1973). Orientation and form. New York: Academic Press. Rock, I. (1974). The perception of disoriented figures. Scientific American, 230, 78-85.

SCAPINELlo, K. F., \& Yarmey, A. D. (1970). The role of familiarity and orientation in immediate and delayed recognition of pictorial stimuli. Psychonomic Science, 21, 329-330.

SERGENT, I. (1984). An investigation into component and configural processes underlying face perception. British Journal of Psychology, 75, 221-242

ShePard, R. N., \& CoOPER, L. A. (1982). Mental images and their transformations. Cambridge, MA: MIT Press.

Shepard, R. N., Metzler, J. (1971). Mental rotation of threedimensional objects. Science, 171, 701-703.

Thompson, P. (1980). Margaret Thatcher-A new illusion. Perception, 9, 483-484.

VAlentine, T. (1986). Encoding processes in face recognition. Doctoral thesis, University of Nottingham, England.

VALENTINE, T., BRUCE, V. (1985). What's up? The Margaret Thatcher illusion revisited. Perception, 14, 515-516.

VAlentine, T., BruCE, V. (1986). The effect of race, inversion and encoding activity upon face recognition. Acta Psychologica, 61, 259-273

YARMEY, A. D. (1971). Recognition memory for familiar "public" faces: Effects of orientation and delay. Psychonomic Science, 24, 286-288

YIN, R. K. (1969). Looking at upside-down faces. Journal of Experimental Psychology, 81, 141-145.

YIN, R. K. (1970). Face recognition by brain-injured patients: A dissociable ability? Neuropsychologia, 8, 395-402.

\section{NOTE}

1. The pictures used were of Elvis Presley, Cliff Richard, Neil Kinnock, Jimmy Saville, and Dennis Waterman. Pictures of Prince Charles and Sean Connery were used in practice trials. These people were chosen because they were the most familiar faces to British students, they were available in a full-face pose, and all had been consistently well recognized in previous experiments.

(Manuscript received June 19, 1987; revision accepted for publication February 25, 1988.) 